\title{
Action intersectorielle sur les déterminants sociaux de la santé et l'équité en santé au Canada : examen des lettres de mandat du gouvernement fédéral de décembre 2019
}

\author{
Kelsey Lucyk, Ph. D.
}

Diffuser cet article sur Twitter

La réduction des inégalités en matière de santé est un défi à l'échelle mondiale, qui nécessite une action intersectorielle sur les déterminants de la santé ${ }^{1}$. Au Canada, le premier ministre fixe aux ministres des objectifs en matière de politiques par l'intermédiaire de " lettres de mandat » : ces lettres décrivent les attentes quant aux fonctions des ministres et déterminent les principales priorités de leur ministère. On peut considérer ces lettres de mandat comme un outil permettant de cerner les possibilités d'action et comme un point de départ dans le processus d'élaboration des politiques. Étant donné que la plupart des déterminants de la santé échappent au secteur de la santé en tant que tel ${ }^{2,3}$, les lettres de mandat adressées aux ministres hors du secteur de la santé révèlent les point d'accès sur lesquels le secteur de la santé peut s'appuyer pour favoriser les politiques contribuant à améliorer l'équité en santé. Notre examen de 33 lettres de mandat permet de définir les principaux engagements hors du secteur de la santé susceptibles d'améliorer les conditions dans lesquelles les gens naissent, vivent, grandissent, travaillent et vieillissent, ce que l'on appelle les déterminants sociaux de la santé ${ }^{4}$. Pour la majorité des engagements relevés dans cet examen, les secteurs autres que celui la santé ne font aucun lien explicite entre leurs directives et la santé ou ses déterminants sociaux, ce qui illustre un défi bien connu et de plus en plus important lié à l'action intersectorielle en matière de santé : la recherche de l'équité en santé est parfois considérée comme une priorité inférieure aux priorités stratégiques d'autres secteurs, voire incompatible avec elles ${ }^{5}$. Notre examen s'adresse aux professionnels en santé publique mais aussi à ceux d'autres secteurs qui travaillent à l'amélioration de l'équité en santé.

\section{Équité en santé et déterminants sociaux de la santé : occasions à saisir}

Dans le cadre de son engagement à un gouvernement ouvert et transparent, le cabinet du premier ministre Justin Trudeau a publié les lettres de mandat du $29^{\text {e }}$ Conseil des ministres du Canada le 13 décembre 20196. Dans plusieurs cas, ces lettres de mandat jettent les bases d'une action intersectorielle sur les déterminants de la santé en précisant quels ministres devraient travailler ensemble, sur quels sujets et à quelles fins. Un exemple digne de mention est la directive donnée en amont à la ministre associée des Finances, visant une intégration des mesures de la qualité de vie dans le processus décisionnel et budgétaire du gouvernement fédéral, grâce à la collaboration avec des collègues des secteurs du développement social et des sciences ${ }^{7}$.

Les domaines thématiques n’ont pas été déterminés a priori dans le cadre de cet examen : nous avons plutôt étudié les lettres en vue de sélectionner les facteurs connus susceptibles de façonner la santé et l'équité en santé (p. ex. la répartition de l'argent, du pouvoir et des ressources) ${ }^{8}$ et nous les avons regroupés en fonction des principaux déterminants, en utilisant comme cadre de référence le cadre de l'Agence de la santé publique du Canada (ASPC) relatif aux déterminants sociaux de la santé ${ }^{4}$. D'autres cadres pertinents (p. ex. les déterminants écologiques, les déterminants

\section{Points saillants}

- Les lettres de mandat des ministres du gouvernement fédéral définissent les possibilités d'action intersectorielle sur les déterminants sociaux de la santé et sur l'équité en santé.

- Les principaux domaines d'action intersectorielle définis dans les lettres de mandat de 2019 sont l'adoption de mesures de bien-être dans le budget fédéral, de politiques fiscales de redistribution et d'initiatives dans les secteurs notamment de l'emploi, du logement et de l'éducation.

- Il est important d'assurer la surveillance continue des inégalités en matière de santé au Canada et d'en rendre compte afin de pouvoir évaluer les progrès et déterminer les secteurs dans lesquels la collaboration intersectorielle peut être renforcée.

Mots-clés : déterminants sociaux de la santé, examen de politiques, équité en santé, santé dans toutes les politiques

commerciaux et les déterminants de la santé spécifiques aux Autochtones) dépassaient la portée de cet examen. Le tableau 1 rassemble les principales constatations à propos des engagements énoncés dans les lettres de mandat quant aux domaines clés des déterminants. Plus précisément, on y trouve reliés les engagements prévus dans les lettres de mandat et les ministres responsables ainsi que ceux 


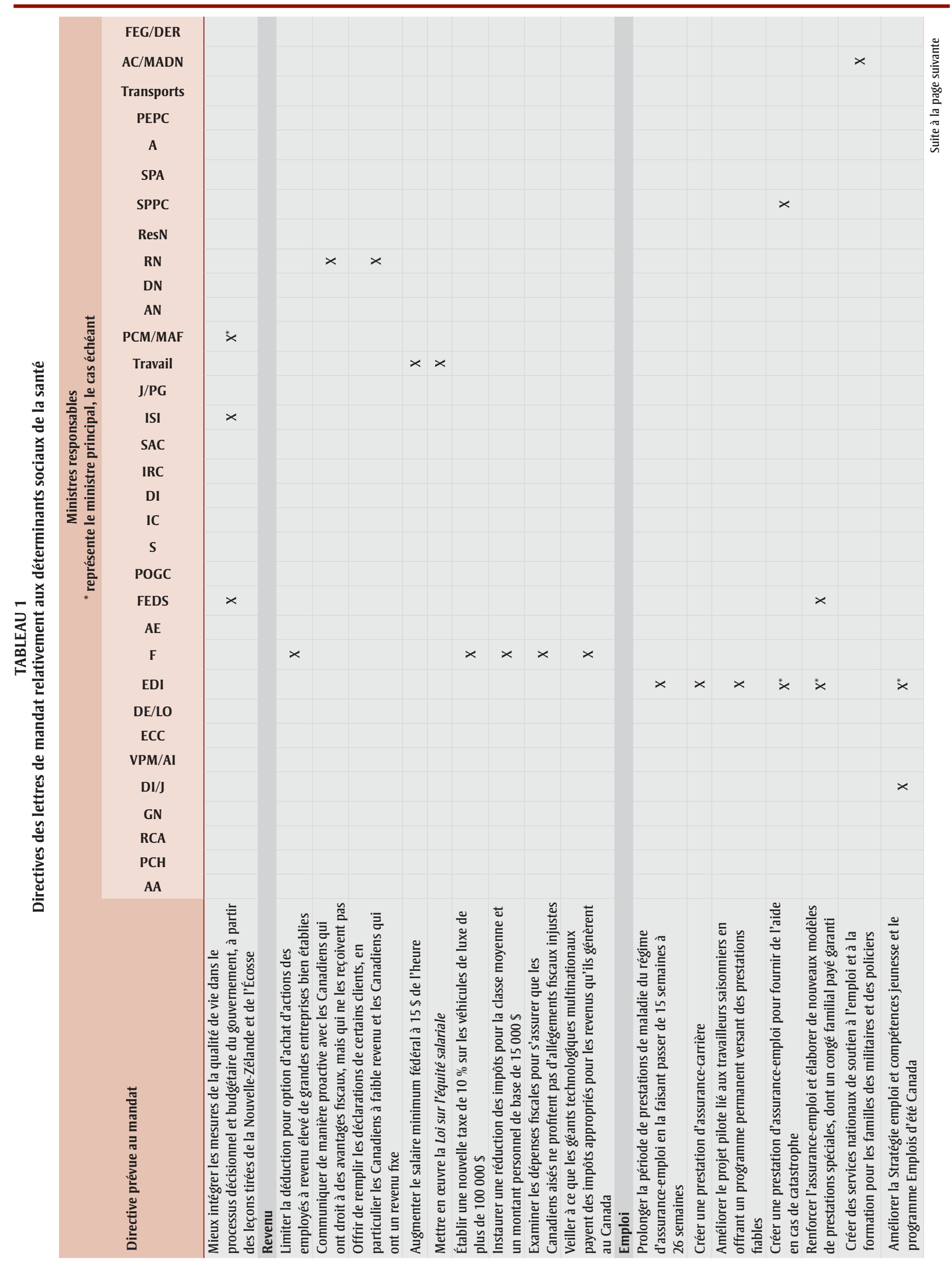




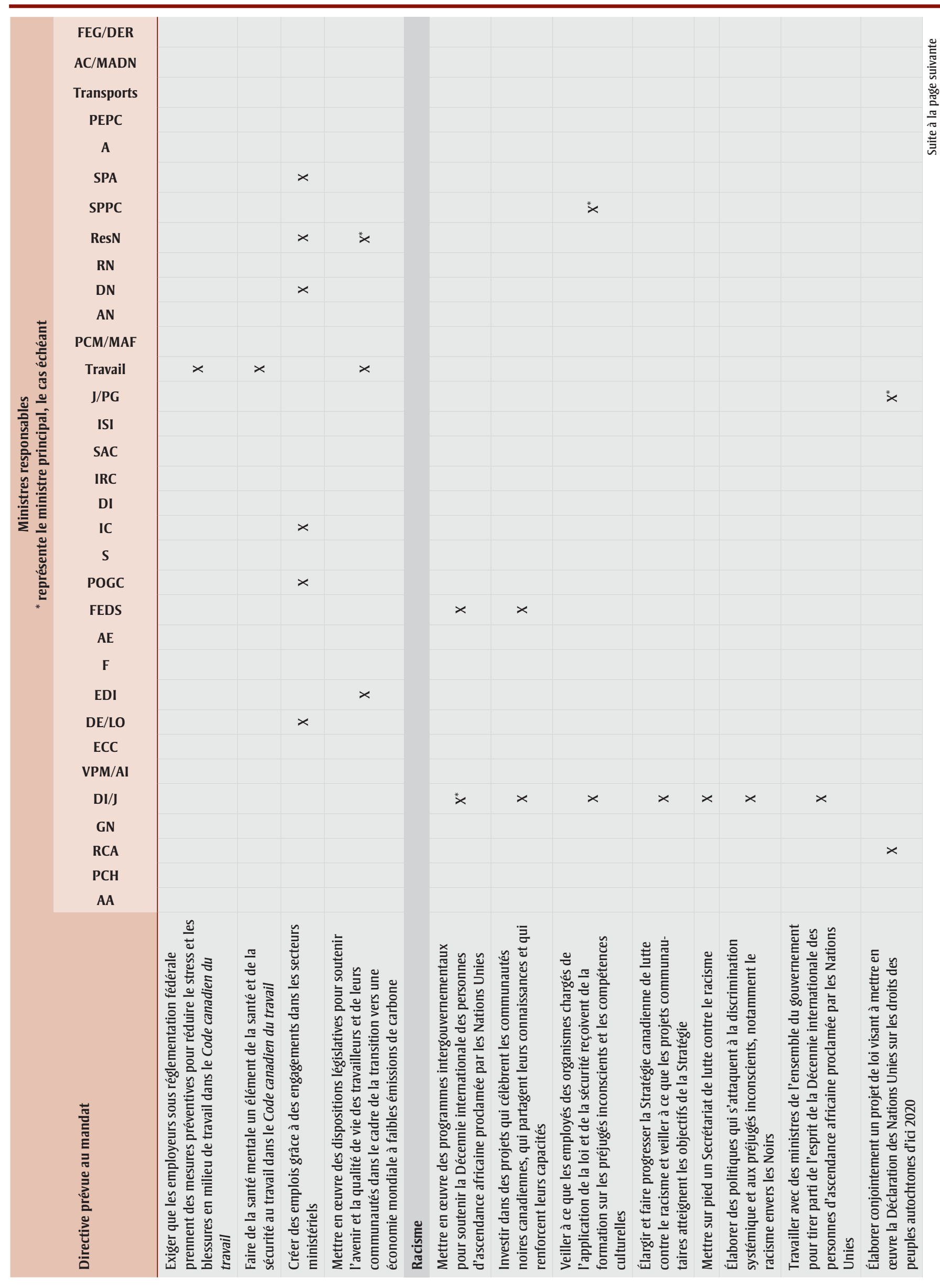




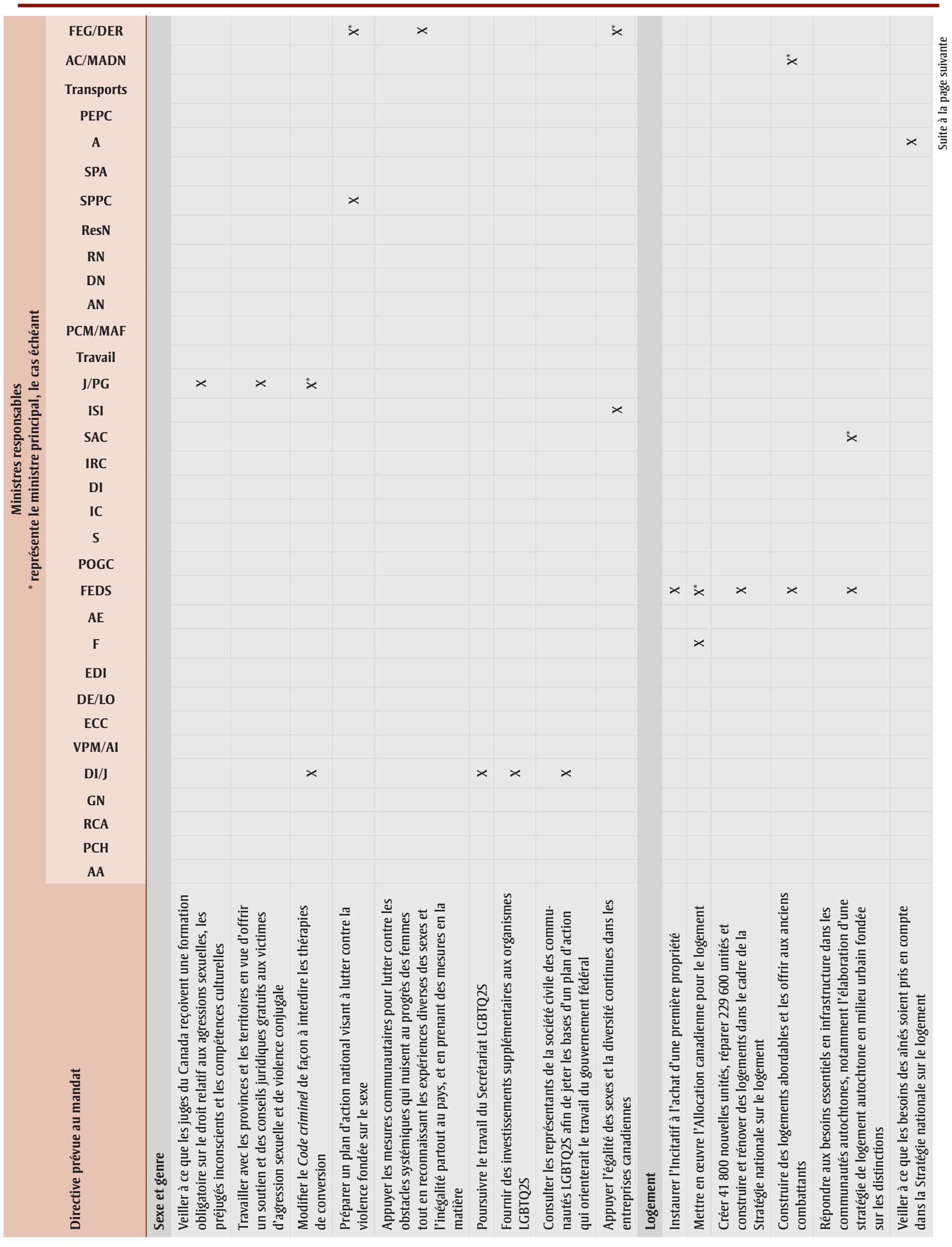




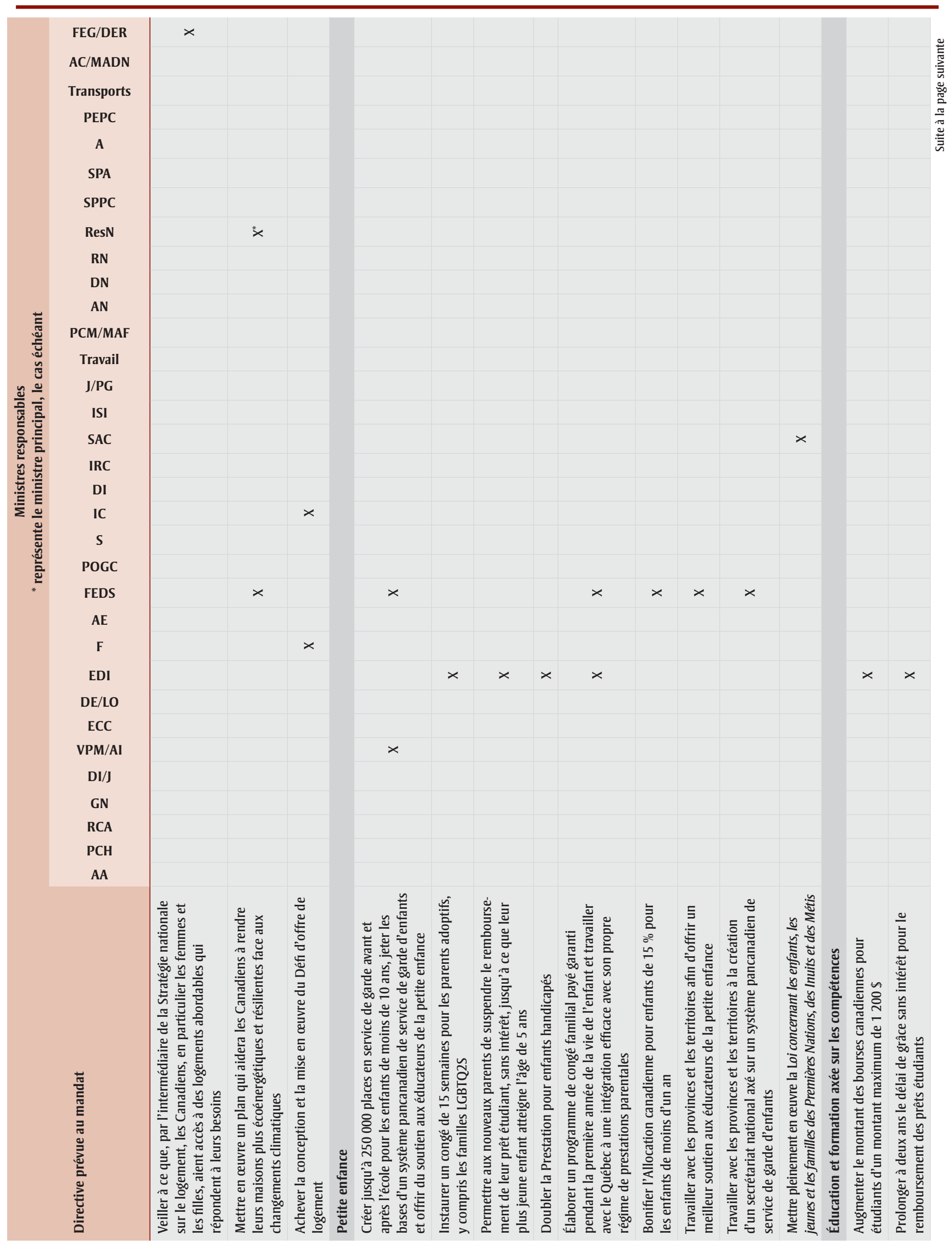




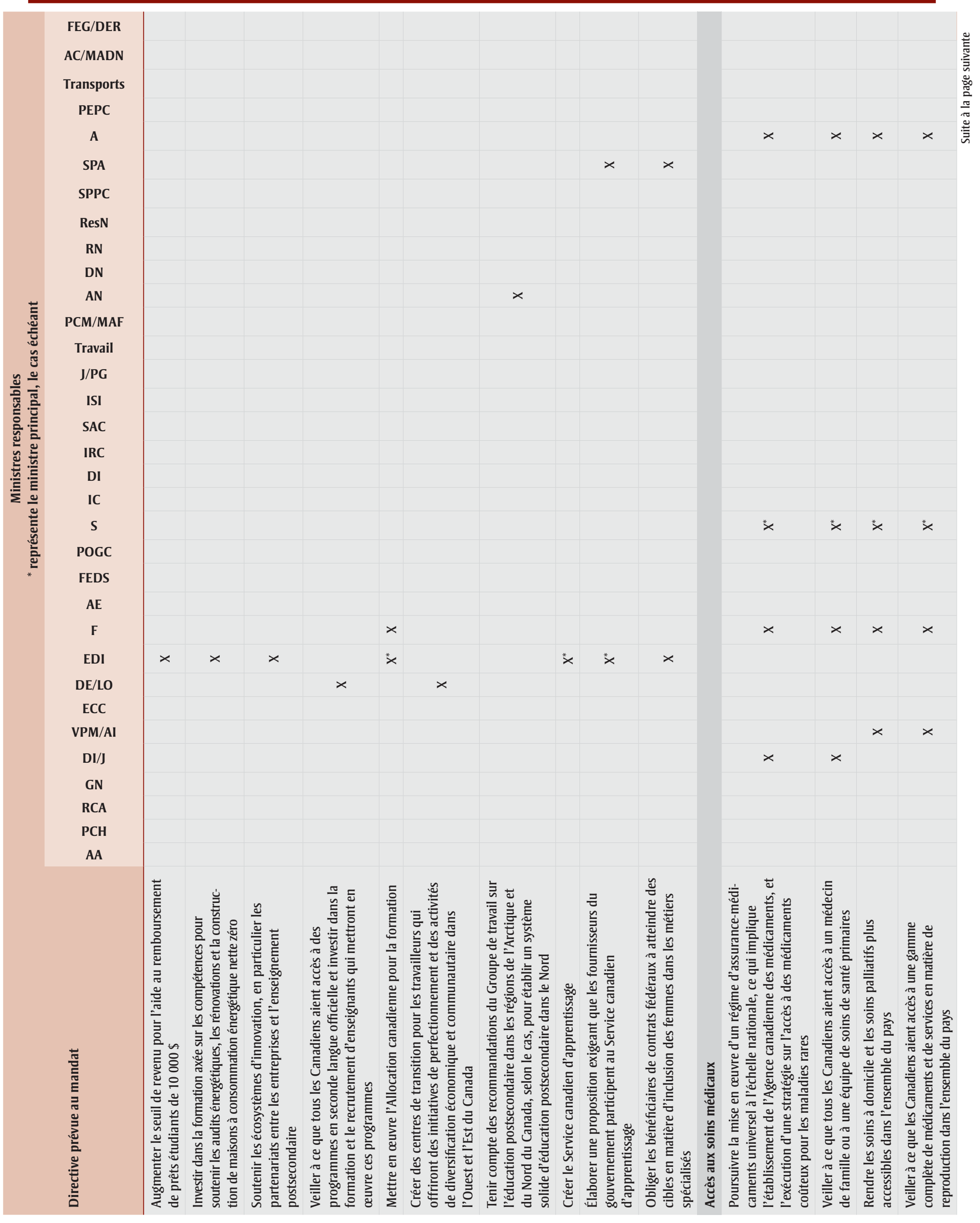




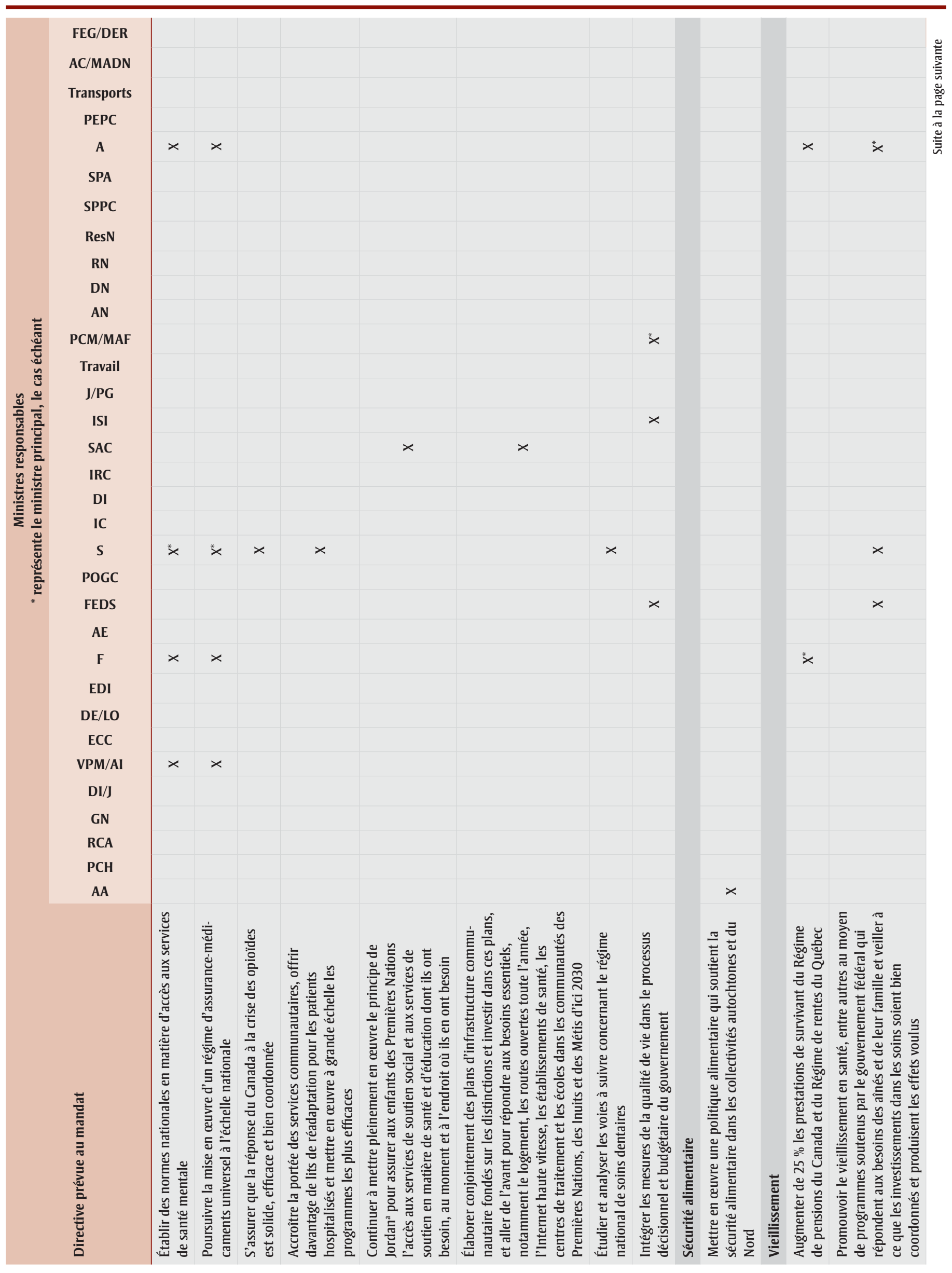




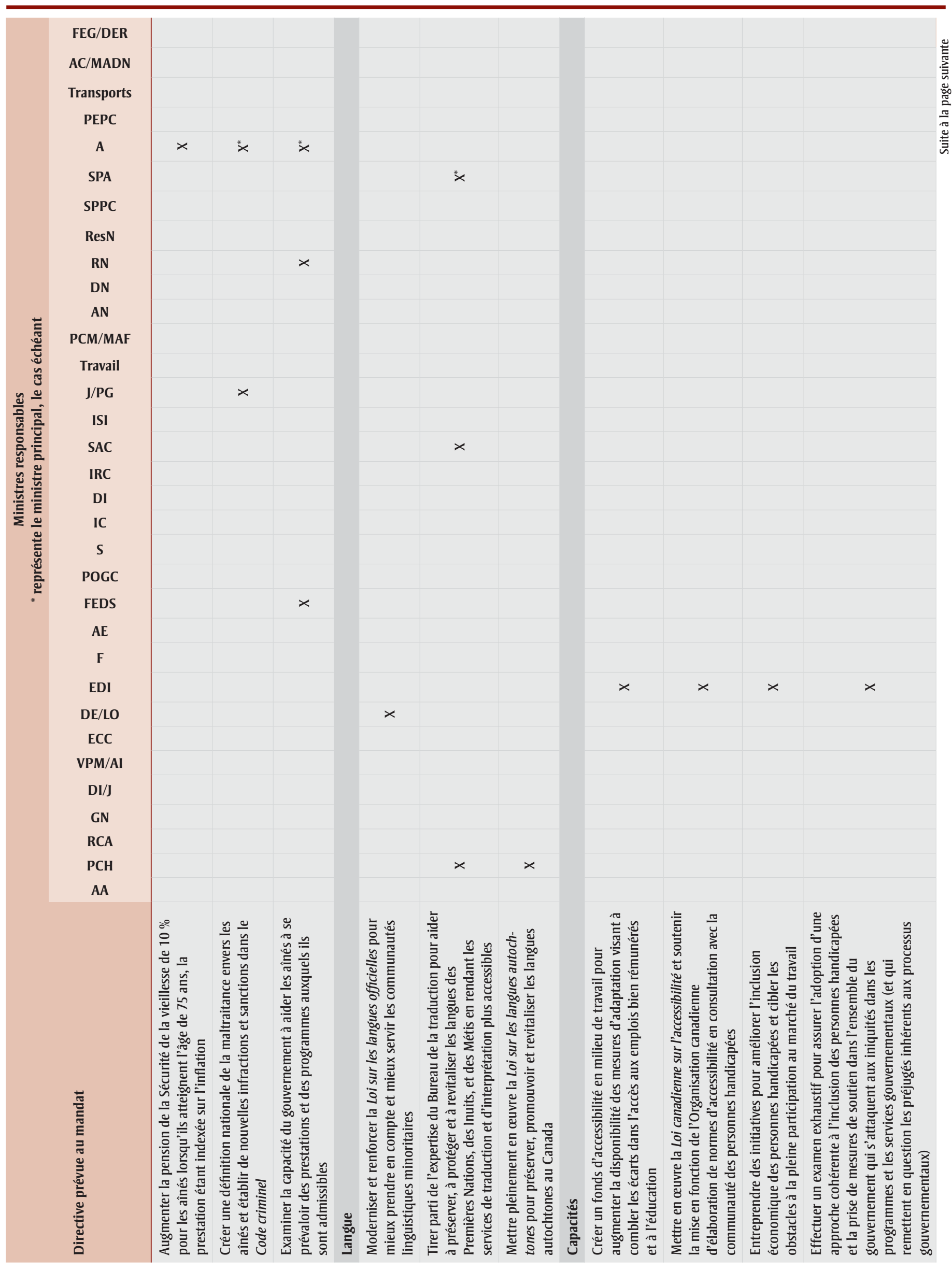




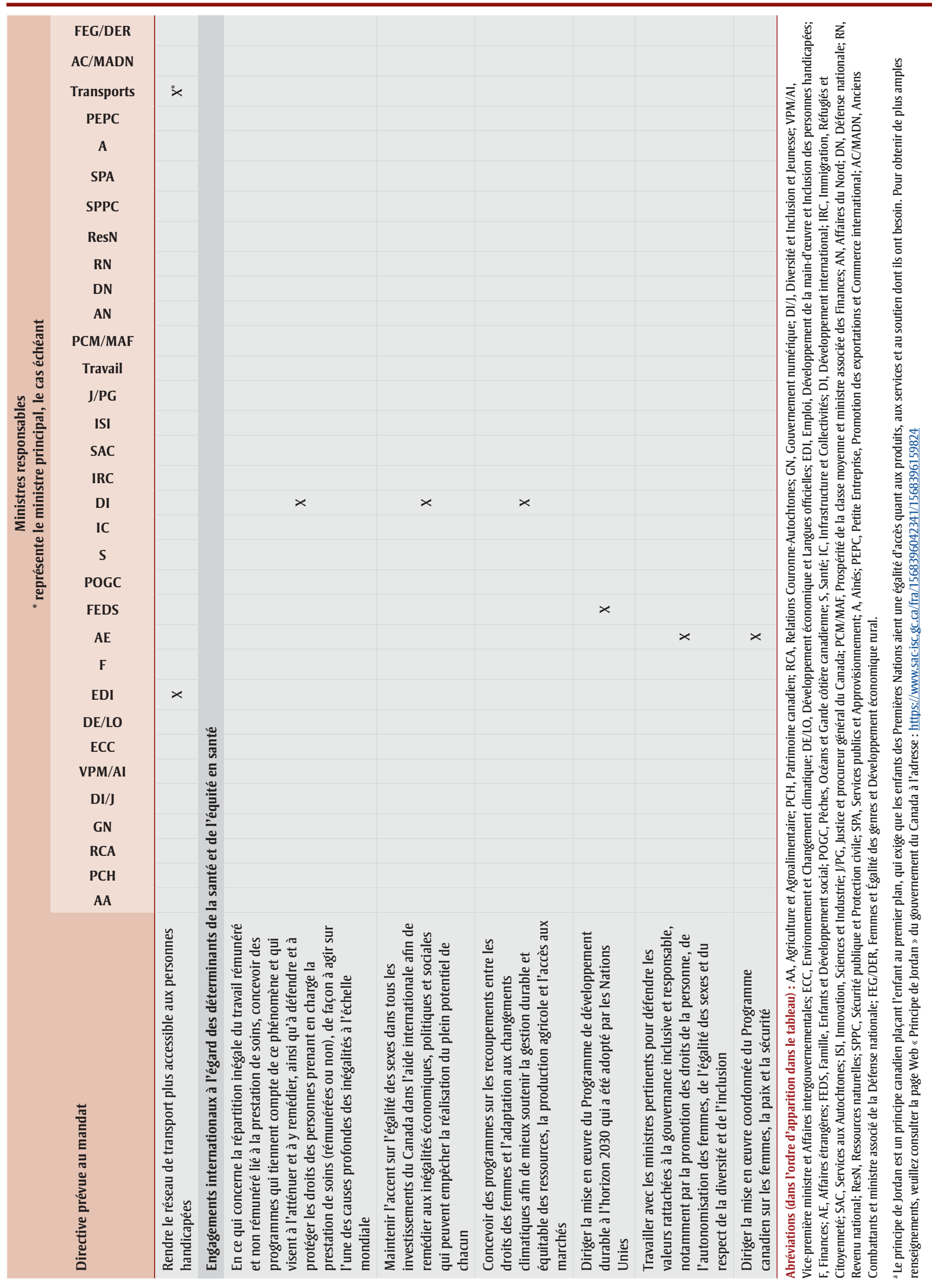


qui sont explicitement nommés dans les engagements.

Les résultats qui suivent visent à montrer la portée et l'ampleur des engagements relatifs aux déterminants sociaux de la santé dans les secteurs autres que la santé mais ne visent pas à évaluer les effets positifs ou négatifs que de telles mesures peuvent avoir sur la santé et les inégalités en matière de santé. Ce rapport est une analyse de 33 des 37 lettres publiées : ont été exclues les lettres adressées au leader du gouvernement à la Chambre des communes, au leader parlementaire adjoint de la Chambre des communes, au président du Conseil privé et au président du Conseil du Trésor. L’analyse a été réalisée entre décembre 2019 et janvier 2020, ce qui signifie que plusieurs politiques ou programmes ont sans doute été mis en œuvre ou réorientés depuis la date de rédaction de cet article (en particulier dans le contexte de la pandémie de COVID-19). Les lettres de mandat, à la fois documents de source primaire et outils stratégiques ambitieux, sont représentatives d'un moment précis, et l'analyse présentée ici décrit les plans d'action intersectorielle à ce moment-là.

\section{Revenu et emploi}

Les politiques fiscales de redistribution et les mesures de soutien supplémentaires (p. ex. sécurité d'emploi et prestations, renforcement du filet de sécurité sociale) sont susceptibles d'améliorer les déterminants de la santé dans la mesure où elles augmentent l'accès aux ressources nécessaires pour maintenir la santé, englobant d'autres déterminants - comme l'emploi ou le revenu -liés à la santé et au bienêtre $^{9}$. Dans le secteur financier, le gouvernement du Canada a proposé d'instaurer une taxe sur les véhicules de luxe et examinera les allégements fiscaux pour s'assurer que les personnes aisées n'en bénéficient pas injustement. Les Canadiens de la classe moyenne devraient bénéficier d'une réduction des impôts et d'une augmentation du montant personnel de base, et le salaire minimum fédéral devrait être porté à au moins 15 \$ de l'heure. En ce qui concerne les aînés, des améliorations devraient être apportées à la pension de la Sécurité de la vieillesse (augmentation de 10 \% à l'âge de 75 ans), ainsi qu'au Régime de pensions du Canada et au Régime de rentes du Québec (augmentation de 25 \% des prestations de survivant). En ce qui concerne les nouveaux parents, l'Allocation canadienne pour enfants devrait être bonifiée pour les enfants de moins d'un an, et la Prestation pour enfants handicapés devrait être doublée. Le gouvernement du Canada tentera également d'aider les aînés et les Canadiens à faible revenu à se prévaloir des prestations et des programmes auxquels ils sont admissibles. Selon la Loi sur l'équité salariale ${ }^{10}$ récemment adoptée et visant à réduire l'inégalité des revenus, les employeurs seront tenus de corriger la discrimination fondée sur le sexe en ce qui concerne la rémunération, afin que les employés reçoivent une rémunération égale pour l'exécution d'un travail de valeur égale dans les catégories d'emploi à prédominance masculine et ceux à prédominance féminine.

Dans le secteur de l'emploi, de nouvelles prestations devraient être offertes aux travailleurs saisonniers et aux employés qui ont perdu leur emploi en raison de la cessation des activités d'un employeur, et des services de soutien à l'emploi devraient être proposés aux familles des militaires et des policiers. Le régime d'assurance-emploi devrait prolonger les prestations de maladie, compenser les pertes de revenu découlant de catastrophes et mettre au point des prestations spéciales pour les nouveaux parents.

\section{Racisme}

Le racisme influe sur la santé à de multiples niveaux, car il réduit l'accès aux déterminants positifs de la santé, augmente l'exposition aux facteurs de risque et a des effets négatifs sur la santé physique et mentale ${ }^{11}$. En ce qui concerne le secteur de la justice pénale, tous les juges du Canada devront suivre une formation sur les préjugés inconscients, et les forces de l'ordre recevront de la formation sur les préjugés inconscients et les compétences culturelles. Des investissements seront réalisés pour célébrer les communautés noires du Canada et renforcer leurs capacités, ainsi que pour soutenir la Décennie internationale des personnes d'ascendance africaine proclamée par les Nations Unies. Plus généralement, la ministre de la Diversité et de l'Inclusion et de la Jeunesse doit élaborer des politiques visant à lutter contre la discrimination systémique et le racisme envers les Noirs. Dans tous les ministères, on s'efforcera de soutenir l'autodétermination, d'améliorer la prestation des services et de faire progresser la réconciliation avec les peuples autochtones, en partie grâce à des mesures législatives mettant en œuvre la Déclaration des Nations Unies sur les droits des peuples autochtones.

\section{Sexe et genre}

Le sexe et le genre (et les concepts connexes) façonnent la santé des personnes et de la population en influençant la répartition des risques pour la santé, des facteurs de protection, de l'accès aux services de santé et d'autres déterminants ${ }^{12}$. Outre les initiatives horizontales, comme l'analyse comparative entre les genres plus et l'analyse de la diversité, plusieurs autres initiatives proposent des améliorations pour les personnes qui ont été désavantagées en raison de leur sexe, de leur genre ou de leur orientation sexuelle. Par exemple, des mesures seront prises pour interdire les thérapies de conversion, grâce à des modifications apportées au Code criminel, et pour accroître la portée et la capacité des organismes LGBTQ2. En ce qui concerne la violence fondée sur le sexe ou le genre, une réponse aux appels à la justice de l'Enquête nationale sur les femmes et les filles autochtones disparues et assassinées sera élaborée et des conseils juridiques gratuits seront fournis aux victimes d'agressions sexuelles et de traumatismes, avec une formation sur ces sujets pour tous les juges canadiens.

\section{Logement}

Le logement est non seulement un déterminant essentiel à la prévention des maladies en soi, mais il est également important en raison de son influence sur d'autres déterminants, comme la stabilité sociale ou l'environnement ${ }^{13}$. Parmi les initiatives visant à améliorer l'abordabilité du logement au Canada figurent l'Allocation canadienne pour le logement et les logements pour les anciens combattants, ainsi que l'Incitatif à l'achat d'une première propriété récemment mis en place. La Stratégie nationale sur le logement permettra de créer plus de 40000 unités, d'en réparer plus de 200000 et de continuer à améliorer l'offre par la construction et la rénovation. Des mesures seront également prises pour garantir que la Stratégie tienne compte des besoins des aînés, des femmes et des filles, et un nouveau plan sera élaboré relativement au logement des Autochtones en milieu urbain. Des solutions novatrices seront étudiées grâce à la mise en place d'un nouveau concours, le Défi d'offre de logement, qui remettra 300 millions de dollars en prix ${ }^{14}$. Un soutien 
sera également fourni aux Canadiens afin de les aider à rendre leurs maisons plus écoénergétiques et résilientes face aux changements climatiques.

\section{Petite enfance}

De la naissance à 6 ans, les enfants vivent une période essentielle sur le plan du développement physique, cognitif, émotionnel et social, qui a un impact sur leur bien-être durant l'enfance et plus tard dans leur vie ${ }^{15}$. Les mesures visant à améliorer la petite enfance comprendront la mise en œuvre d'un nouveau congé parental pour les parents adoptifs et un congé payé garanti pendant la première année de vie de l'enfant. Les nouveaux parents pourront également suspendre le remboursement de leur prêt étudiant jusqu'à ce que leur plus jeune enfant atteigne l'âge de 5 ans. Jusqu'à 250000 nouvelles places en service de garde avant et après l'école seront créées pour les enfants de moins de 10 ans, et les bases d'un système pancanadien de services de garde d'enfants seront jetées. En ce qui concerne les communautés autochtones, une nouvelle mesure législative sur la protection de l'enfance entrera en vigueur : elle permettra aux communautés d'élaborer des politiques et des lois sur les services à l'enfance et à la famille, en fonction de leur histoire, de leur culture et de leur situation particulières.

\section{Éducation et formation axée sur les compétences}

L'éducation et ses déterminants connexes (comme les compétences) sont susceptibles de façonner les déterminants de la santé en influant sur les possibilités d'emploi, la prise de décision, la position sociale et divers autres éléments ${ }^{16}$. Pour améliorer l'abordabilité de l'enseignement postsecondaire, les montants du Programme canadien de bourses aux étudiants et le seuil de revenu pour l'aide au remboursement de prêts étudiants seront augmentés. Des efforts seront déployés pour garantir que les étudiants inuits, métis et des Premières Nations, ainsi que les étudiants des régions du Nord et de l'Arctique, obtiennent du soutien pour accéder à l'enseignement postsecondaire et réussir leurs études. Un nouveau crédit d'impôt remboursable sera instauré pour les travailleurs canadiens qui suivent une formation, et des centres de transition pour les travailleurs seront créés en vue de soutenir le développement dans l'Ouest et
l'Est du Canada. Enfin, un Service canadien d'apprentissage sera créé pour que les apprentis Sceau rouge aient suffisamment d'occasions d'acquérir l'expérience de travail qui leur est nécessaire. L'expérience de travail pour les jeunes sera soutenue par des améliorations apportées à la Stratégie emploi et compétences jeunesse et au programme Emplois d'été Canada. La création d'emplois est à la base de nombreuses initiatives gouvernementales (p. ex. projets d'infrastructure, la construction navale, les nouvelles technologies).

\section{Engagements internationaux à l'égard des déterminants de la santé et de l'équité en santé}

Étant donné que les inégalités en matière de santé proviennent d'une répartition inégale de l'argent, du pouvoir et des ressources au sein des nations et entre elles ${ }^{8}$, il est important d'examiner comment les investissements stratégiques internationaux du Canada tiennent compte des déterminants de la santé. Dans le cadre de l'aide et des activités internationales, l'accent sur l'égalité des sexes sera maintenu et l'autonomisation des femmes soutenue, par exemple en offrant des possibilités de réduction de la pauvreté chez les femmes dans les pays en développement, en réduisant les disparités salariales dans le domaine des soins et en mettant en œuvre le Programme canadien sur les femmes, la paix et la sécurité. La mise en œuvre du Programme de développement durable à l'horizon 2030 des Nations Unies va se poursuivre. Ce programme comprend de nombreux objectifs liés aux déterminants de la santé, notamment l'éradication de la faim et de la pauvreté et la réduction des inégalités ${ }^{17}$. Des programmes seront élaborés pour soutenir un développement international durable et équitable qui tient compte des recoupements entre les droits des femmes et l'adaptation aux changements climatiques.

\section{Nouvelles orientations pour la recherche en santé}

À la lumière de cet examen, il convient également d'envisager le développement de nouveaux travaux de recherche favorisant l'équité en santé et les déterminants de la santé. En matière de recherche en santé, un Institut national de recherche sur la santé des femmes sera créé pour combler les lacunes en matière de recherche et de soins, avec l'adoption d'une approche intersectionnelle. Le Conseil de recherches en sciences humaines et les Instituts de recherche en santé du Canada vont créer des bourses de recherche pour des études sur la race, la diversité et le genre. Hors du secteur de la santé, la ministre de la Diversité et de l'Inclusion et de la Jeunesse va investir dans la recherche sur les nouvelles arrivantes appartenant à une minorité visible. Enfin, le Conseil national de recherches du Canada mènera des travaux de recherche sur des enjeux comme les changements climatiques, une croissance propre et une société saine facteurs qui façonnent les conditions de santé.

Notre examen a porté sur les lettres de mandat du gouvernement fédéral, que l'on peut considérer à la fois comme une structure de gouvernance et comme une mesure de gouvernance pour l'équité en santé, du point de vue de la "santé dans toutes les politiques $»^{18}$. En tant que structure, les lettres facilitent la collaboration sur des initiatives particulières qui peuvent avoir un impact sur la santé ou l'équité en santé, grâce au regroupement des acteurs intersectoriels (c.-à-d. les ministres). En tant que mesure, les lettres contribuent à l'élaboration des politiques par l'établissement de programmes ministériels et par la définition des objectifs à atteindre pendant le mandat. Toutefois, les objectifs énoncés dans les lettres de mandat ne sont pas contraignants et, bien que le gouvernement fédéral assure un suivi des engagements pour obtenir des résultats stratégiques concrets ${ }^{19}$, il faut approfondir l'analyse pour déterminer le rôle des lettres de mandat dans la réalisation de ces mesures tout au long du processus d'élaboration des politiques. Cette analyse peut porter sur la manière dont les engagements en dehors du secteur de la santé sont reliés aux améliorations en matière de santé de la population à court et à long terme, ainsi que sur la détermination des secteurs qui font preuve de leadership et d'efficacité dans la mise en œuvre des initiatives intersectorielles. Elle peut également consister à étudier la façon dont les engagements évoluent entre la création et la mise en œuvre, et observer quels facteurs mènent à une mise en œuvre à long terme dans un contexte où priorités, mandats, gouvernements et divers facteurs contextuels sont fluctuants.

\section{Conclusion}

Notre examen a mis en évidence un large éventail de secteurs dans lesquels divers 
ministères fédéraux vont agir afin d'atteindre les objectifs communs de bien-être social, sanitaire et économique, en intervenant sur les principaux déterminants sociaux de la santé à l'aide du cadre de l'ASPC 4 . La collaboration et les partenariats intersectoriels portant sur des déterminants comme le revenu, l'emploi, le racisme et autres sont essentiels pour améliorer l'équité en santé, la collaboration étant un message clé dans toutes les lettres de mandat pour atteindre la réalisation des progrès. L'ASPC s'attèle à ces travaux grâce à ses partenariats avec le gouvernement et avec d'autres intervenants, grâce à ses investissements auprès des populations qui subissent des inégalités en matière de santé (p. ex. les Canadiens noirs, les peuples autochtones) et grâce à ses efforts soutenus de mesure des inégalités en matière de santé au Canada et de rapports sur le sujet ${ }^{20}$. Cependant, comme notre examen le révèle, il existe d'autres occasions où des initiatives hors du secteur de la santé peuvent être explorées et mises à profit de façon à améliorer l'équité en santé. Les leçons tirées d'approches prometteuses en cours dans d'autres administrations (p. ex. "santé dans toutes les politiques ", évaluation des répercussions) vont continuer d'être suivies avec beaucoup d'intérêt afin d'éclairer les efforts visant à atteindre l'équité en santé grâce à des mesures intersectorielles.

\section{Remerciements}

Je tiens à souligner le travail de ceux qui se consacrent à l'équité en santé au Canada et à l'étranger, notamment Nancy Gehlen, Grace Wan Te et des collègues de l'équipe des initiatives et des partenariats intersectoriels de l'Agence de la santé publique du Canada, qui ont fourni des commentaires réfléchis et un appui à la rédaction des premières versions de ce commentaire.

\section{Conflits d'intérêts}

Aucun.

\section{Contribution de l'auteure et avis}

Kelsey Lucyk est responsable de la conception, de l'analyse et de la rédaction de l'article. Le contenu de l'article et les points de vue qui y sont exprimés n'engagent qu'elle : ils ne correspondent pas nécessairement à ceux du gouvernement du Canada.

\section{Références}

1. Organisation mondiale de la santé L'équité en santé grâce à l'action intersectorielle : analyse d'études de cas dans 18 pays. Ottawa (Ont.) : Agence de la santé publique du Canada; 2008 [N $\mathrm{N}^{\circ}$ au catalogue : HP5-67/2008F]. 56 p. En ligne à : https://www.who .int/social_determinants/resources /health_equity_isa_2008_fr.pdf

2. World Health Organization (WHO). Intersectoral action [Internet]. Genève (CH) : WHO; 2020 [consultation le 7 janvier 2020]. Consultable en ligne à la page : https://www.who.int /social_determinants/thecommission /countrywork/within/isa/en/

3. Sous-comité sénatorial sur la santé des populations. Un Canada en santé et productif : une approche axée sur les déterminants de la santé. Ottawa (Ont.) : Sénat du Canada; 2009. 61 p.

4. Agence de la santé publique du Canada. Déterminants sociaux de la santé et inégalités en santé [Internet]. Ottawa (Ont.) : Gouvernement du Canada; 2019 [consultation le 16 janvier 2020]. En ligne à : https://www.canada.ca/fr/sante -publique/services/promotion-sante /sante-population/est-determine-sante .html

5. Smith M, Weinstock D. Reducing health inequities through intersectoral action: balancing equity in health with equity for other social goods. Int J Health Policy Manag. 2019;8(1):1-3.

6. Gouvernement du Canada. Lettres de mandat [Internet]. Ottawa (Ont.) : Gouvernement du Canada; 2019 [consultation le 7 janvier 2020]. En ligne à : https://pm.gc.ca/fr/lettres-de-mandat

7. Cabinet du premier ministre. Lettre de mandat de la ministre de la Prospérité de la classe moyenne et ministre associée des Finances [Internet]. Ottawa (Ont.) : Gouvernement du Canada; 2019. En ligne à : https:// pm.gc.ca/fr/lettres-de-mandat/2019 /12/13/lettre-de-mandat-de-la-ministre -de-la-prosperite-de-la-classe-moyenne

8. Commission des déterminants sociaux de la santé. Combler le fossé en une génération : Instaurer l'équité en santé en agissant sur les déterminants sociaux de la santé. Genève $(\mathrm{CH})$ : Organisation mondiale de la santé; 2008. 260 p. En ligne à : https://www.who.int/social_ determinants/thecommission/finalreport /fr/
9. Raphael D (dir.). Social determinants of health. 2e éd. Toronto (Ont.) : Canadian Scholars' Press Inc; 2009. $495 \mathrm{p}$.

10. Loi sur l'équité salariale (L.C. 2018, ch. 27, art. 416). Ottawa (Ont.) : Gouvernement du Canada. En ligne à : https://laws-lois.justice.gc.ca/fra /lois/P-4.2/page-1.html

11. Paradies Y, Ben J, Denson N, et al. Racism as a determinant of health: a systematic review and meta-analysis. PLoS One [Internet]. 2015 [consultation le 6 mai 2020];10(9):e0138511. En ligne à : https://www.ncbi.nlm.nih .gov/pmc/articles/PMC4580597/\# _ffn_sectitle

12. Administrateur en chef de la santé publique. Rapport sur l'état de la santé publique au Canada 2012 : Le sexe et le genre - Leur influence importante sur la santé. Ottawa (Ont.) : Agence de la santé publique du Canada; 2012 [ $\mathrm{N}^{\mathrm{o}}$ au catalogue : HP2-10/2012F]. 175 p.

13. Centre de collaboration nationale des déterminants de la santé. Le logement, une cible prioritaire de la santé publique en matière d'équité : liste de lectures essentielles [Internet]. Antigonish (N.-É.) : Université St Francis Xavier; 2018 [consultation le 6 mai 2020]. En ligne à : http://nccdh.ca/fr/resources /entry/housing-as-a-focus-for-public -health-action-on-equity-a-curated-list

14. Impact Canada. Défi d’offre de logement [Internet]. Ottawa (Ont.) : Gouvernement du Canada; 2019 [consultation le 8 janvier 2020]. En ligne à : https://impact.canada.ca/fr /defis/defi-doffre-de-logement

15. Conseil canadien des déterminants sociaux de la santé (CCDSS). Mise en œuvre d'initiatives multisectorielles de développement sain durant l'enfance : leçons tirées d'interventions communautaires [Internet]. Ottawa (Ont.) : CCDSS; 2017. En ligne à : http://ccsdh.ca/images/uploads/FR_ Implementing_Multi-Sectoral_HCD_ Initiatives.pdf

16. Low B, Low D. Education and education policy as social determinants of health. Virtual Mentor. 2006;8(11): 756-761. doi:10.1001/virtualmentor .2006.8.11.pfor1-0611. 
17. Nations Unies. Le programme de développement durable [Internet]. New York (NY) : Nations Unies; 2015 [consultation le 7 janvier 2020]. En ligne à : https://www.un.org / sustainabledevelopment/fr /development-agenda/

18. McQueen D, Wismar M, Lin V, Jones C, Davies M (dir.). Intersectoral governance for health in all policies: structures, actions, and experiences [Internet]. Copenhagen (Denmark) : WHO on behalf of the European Observatory on Health Systems and Policies; 2012. 221 p. En ligne à : http://www.euro.who.int/__data /assets/pdf_file/0005/171707 /Intersectoral-governance-for-health -in-all-policies.pdf

19. Bureau du Conseil privé. Suivi des lettres de mandat : livrer des résultats pour les Canadiens [Internet]. Ottawa (Ont.) : Gouvernement du Canada; 2020 [consultation le 5 mai 2020]. En ligne à : https://www.canada.ca/fr /conseil-prive/campagnes/mandat -suivi-resultats-canadiens.html

20. Agence de la santé publique du Canada. Les principales inégalités en santé au Canada : un portrait national [Internet]. Ottawa (Ont.) : Agence de la santé publique du Canada; 2018 [consultation le 7 janvier 2020]. En ligne à : https://www.canada.ca/fr /sante-publique/services/publications /science-recherche-et-donnees/rapport -principales-inegalites-sante-canada -sommaire-executif.html 